\title{
Prediction of individual response to postnatal dexamethasone in ventilator dependent preterm infants
}

\author{
Carl Kuschel, Nick Evans, Albert Lam
}

\begin{abstract}
Aims-To evaluate factors predictive of individual response to dexamethasone in preterm infants.

Methods-A cohort of 74 preterm infants born between January 1993 and February 1996 was studied retrospectively. All of them had received dexamethasone to facilitate weaning from artificial ventilation. Demographic factors, ventilation parameters, and details of dexamethasone administration were recorded from the medical and nursing notes. Radiographs were assessed by one observer who was unaware of the clinical condition of the infant or the outcome. Outcome variables examined included change in ventilation index (VI) at 36-48 hours, the number of days to extubation from the start of dexamethasone, and death before extubation.
\end{abstract}

Results-Most babies improved but changes in VI at 36-48 hours ranged from substantial deterioration to dramatic improvement. No identifiable factors were significantly associated with this range of response. The median time to extubation was 6 days. The 36 babies who extubated within the first 6 days were: significantly more mature; less likely to have pulmonary interstitial emphysema (PIE) or pneumothorax; and had significantly lower VIs in the 12 hours preceding dexamethasone treatment. The postconceptional age at extubation was the same whether babies were extubated within or after the first 6 days. Multiple linear regression confirmed a significant association between number of days to extubation and the three factors described above (adjusted $\left.R^{2}=0.5126\right)$.

Conclusions-Individual responses to dexamethasone can be partly predicted by gestation, the presence of PIE, and the VI before dexamethasone administration. (Arch Dis Child Fetal Neonatal Ed 1998;78:F199-F203)

Keywords: chronic lung disease; dexamethasone; pulmonary interstitial emphysema

Several randomised controlled trials have shown that dexamethasone reduces the duration of ventilatory support in preterm infants who are developing chronic lung disease (CLD).$^{1-8}$ It has been our clinical observation that there is a wide range of individual responses to postnatal corticosteroids, with some infants benefiting rapidly, with a marked decrease in their ventilatory requirement while others scarcely improve or even deteriorate despite treatment. Dexamethasone produces side effects, notably glucose intolerance, ${ }^{246}$ cardiac hypertrophy, ${ }^{9}$ gastrointestinal complications ${ }^{10}$ and it affects growth. ${ }^{26}$ It is important, therefore, to predict those babies least likely to benefit from postnatal steroids.

This study aimed to examine the range of responses and the factors which predicted the response in a cohort of infants receiving dexamethasone to facilitate weaning from artificial ventilation. We hypothesised, on the basis of our clinical observations, that infants who were less likely to extubate rapidly would be of lower gestation and more likely to have radiological features of pulmonary interstitial emphysema (PIE).

\section{Methods}

Study infants were identified from a computer database, specifically searching for those preterm infants who had received dexamethasone. To ensure that all cases were identified, a secondary search was undertaken to identify those infants who had chronic lung disease (CLD) who required ventilation for more than 10 days, or those infants who died before 10 days. The search was confined to infants who were born between 1 January 1993 and 29 February 1996. The notes of all eligible infants were then reviewed in detail. Data were collected prospectively on several infants born towards the end of the study period.

Demographic details were collected regarding the circumstances surrounding delivery of the infant, including antenatal corticosteroid administration, mode of delivery, and reason for delivery. Neonatal details were collected, including gestation in completed weeks (calculated from the certain date of the last menstrual period, or from the best available estimate by ultrasound scan), birthweight, gender, Apgar scores, the use of surfactant, the incidence of pneumothorax, and the use of inotropes and vasodilators in the first 72 postnatal hours. For all blood gas samples, a ventilation index (VI) was calculated using the formula:

$$
\mathrm{VI}=\frac{\mathrm{PaCO}_{2} \times \mathrm{RR} \times \mathrm{PIP}}{1000}
$$

where $\mathrm{PaCO}_{2}$ represents the arterial carbon dioxide tension ( $\mathrm{mm} \mathrm{Hg}$ ), $\mathrm{RR}$ represents the ventilator respiratory rate (breaths per minute), and PIP represents the peak inspiratory pressure $\left(\mathrm{cm} \mathrm{H}_{2} \mathrm{O}\right)$. Blood gas measurements 
Table 1 Characteristics of study cohort (values are expressed as median with range or number and percentage) $n=74$

\begin{tabular}{lc}
\hline Gestation (weeks) & $26(24-31)$ \\
Birthweight $(\mathrm{g})$ & $835(539-1845)$ \\
Males & $47(64 \%)$ \\
Antenatal steroids (>24 hours before & \\
$\quad$ delivery) & $45(61 \%)$ \\
Apgar score at 5 minutes & $7(1-9)$ \\
Primary respiratory diagnosis & \\
$\quad$ Respiratory distress syndrome & $56(76 \%)$ \\
Normal/immature lungs & $12(16 \%)$ \\
Congenital pneumonia & $2(3 \%)$ \\
Other & $4(5 \%)$ \\
Number receiving surfactant & $59(80 \%)$ \\
Pneumothorax requiring intercostal drain & $9(12 \%)$ \\
PDA requiring indomethacin or ligation & $46(63 \%)$ \\
Age at start of dexamethasone (days) & $11(3-31)$ \\
Length of dexamethasone course (days) & $24(3-57)$ \\
\hline
\end{tabular}

were recorded for the first 72 hours after birth, and the 24 hours preceding and 120 hours following the start of treatment with dexamethasone. Because of the variable intervals at which blood gas samples were taken for each baby, VI values were grouped into 12 hourly blocks and then averaged to give a mean VI for that time period. The short term response was assessed by expressing the mean VI for the period 36-48 hours following dexamethasone administration as a percentage of the VI in the 12 hours preceding dexamethasone.

The chest radiograph before the start of dexamethasone was assessed by a paediatric radiologist (AL) masked to all clinical details. Radiographs were graded according to the Northway classification ${ }^{11}$; the presence or absence of any degree of PIE was noted.

Details of dexamethasone administration were noted. Dexamethasone is generally indicated towards the end of the first week of life if the infant has made little progress in weaning from positive pressure ventilation. Our nursery used a 42 day course of dexamethasone at a starting dose of $0.5 \mathrm{mg} / \mathrm{kg} /$ day. ${ }^{1}$ However, significant adverse effects were more common with this course of treatment which often led to a reduction of the course length. Since December 1995, our nursery has used a 14 day course at a starting dose of $0.5 \mathrm{mg} / \mathrm{kg} / \mathrm{day}$, with the course length being extended at the discretion of the attending neonatologist. Clinical events that could be considered to be complications of dexamethasone administration were also recorded during data collection.
The number of days to extubation from the start of treatment were recorded. In the event that the infant died before extubation the circumstances surrounding this were noted. A consistent unit extubation protocol is adhered to by all the senior clinicians which aims for early extubation to nasal continuous positive airways pressure (CPAP) when infants have been stable for up to 24 hours in low concentrations of oxygen with a PIP of 16 to 18 $\mathrm{cm} \mathrm{H}_{2} \mathrm{O}$ and a rate of 10 breaths per minute. It is very unusual for babies to be left on low rate ventilation for more than 24 hours. Infants below 30 weeks of gestation are given aminophylline to facilitate extubation.

Data were analysed using a $\chi^{2}$ test, MannWhitney U test, and multiple linear regression. Statistics were performed the Statistical Package for the Social Sciences (SPSS for Windows, Release 6.1, SPSS Inc., June 1994).

\section{Results}

Seventy six preterm infants received dexamethasone to assist with weaning from artificial ventilation. One infant who received it to treat post-extubation laryngeal oedema was excluded, as was one infant who was receiving care in another hospital. Radiographs were located for 67 of the 74 study infants $(91 \%)$. The characteristics of the entire cohort are shown in table 1.

\section{RESPONSE AT 36-48 HOURS}

The VI at 36-48 hours as a percentage of the pre-dexamethasone VI was examined. The range of response varied from extubation before 36-48 hours (assigned a value of $0 \%$ ) to marked worsening of the VI $(173 \%$ of the preDexamethasone VI). Infants were arbitrarily divided into two groups on the basis of their response - one group who had either worsening or less than a $10 \%$ improvement in the VI at $36-48$ hours $(n=11,15 \%)$, and another group who made up the remainder of the infants ( $\mathrm{n}=$ $61,85 \%)$. This cutoff was used as it was felt that the group with the least response would be that in which clinicians would be interested.

Univariate comparisons of the two groups were performed (table 2). While there were trends to higher rates of PIE, pneumothorax, patent ductus arteriosus and more progressive

Table 2 Comparisons of infants with more than a $10 \%$ improvement with those who had a minimal response in VI at 36-48 hours (values are expressed as median with range or number and percentage)

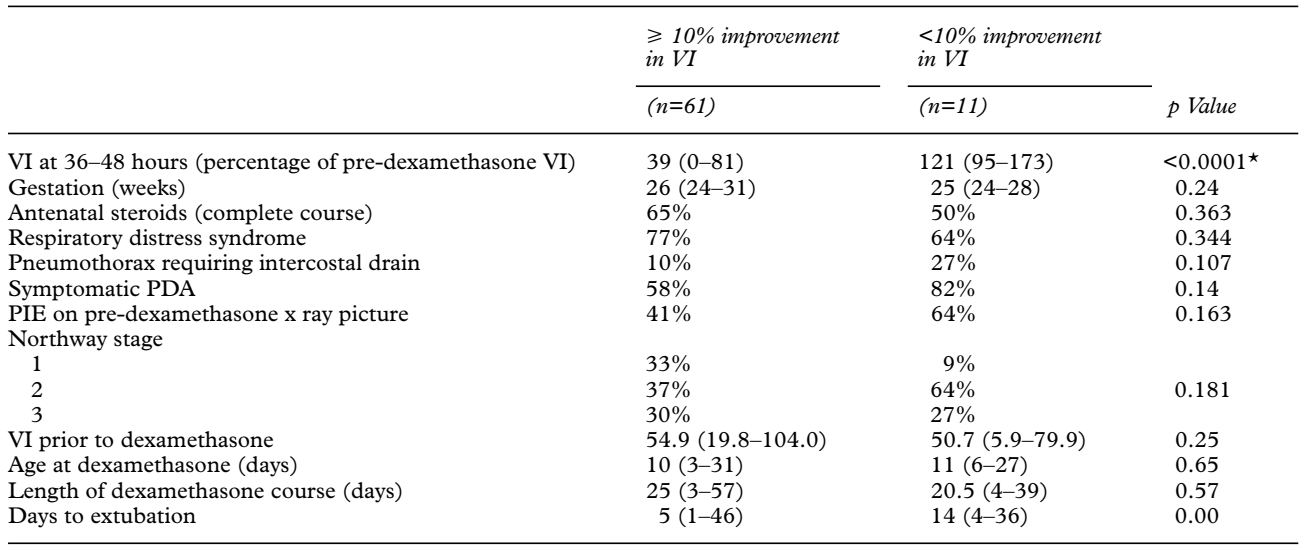


Table 3 Characteristics of infants grouped according to extubation within one week of dexamethasone (values are expressed as median with range or number and percentage)

\begin{tabular}{|c|c|c|c|}
\hline & $\begin{array}{l}\text { Extubation } 6 \text { days } \\
\text { or less }\end{array}$ & $\begin{array}{l}\text { Extubation } 7 \text { days } \\
\text { or more }\end{array}$ & \\
\hline & $(n=36)$ & $(n=33)$ & $p$ Value \\
\hline Gestation (weeks) & $26(24-31)$ & $25(24-29)$ & 0.0017 \\
\hline Birthweight $(\mathrm{g})$ & $925(539-1845)$ & $810(560-1260)$ & 0.03 \\
\hline Antenatal steroids (complete course) & $66 \%$ & $61 \%$ & 0.78 \\
\hline Respiratory distress syndrome & $81 \%$ & $80 \%$ & 0.44 \\
\hline Pneumothorax requiring intercostal drain & $3 \%$ & $21 \%$ & 0.01 \\
\hline Symptomatic PDA & $51 \%$ & $70 \%$ & 0.123 \\
\hline PIE on pre-dexamethasone $\mathrm{x}$ ray picture & $13 \%$ & $72 \%$ & $<0.0001$ \\
\hline \multicolumn{4}{|l|}{ Northway stage } \\
\hline 1 & $47 \%$ & $13 \%$ & \\
\hline 2 & $23 \%$ & $56 \%$ & 0.005 \\
\hline 3 & $30 \%$ & $31 \%$ & \\
\hline VI before dexamethasone & $46.8(5.9-88.5)$ & $60.9(19.8-104.0)$ & 0.02 \\
\hline Age at dexamethasone (days) & $11.5(6-31)$ & $10(3-31)$ & 0.06 \\
\hline Length of course (days) & $19(3-43)$ & $30(3-57)$ & 0.0903 \\
\hline VI at 36-48 hours (percentage of pre-dexamethasone VI) & $33(0-74)$ & $58(18-173)$ & $<0.0001$ \\
\hline Days to extubation & $3(1-6)$ & $14.5(7-46)$ & $<0.0001$ \\
\hline Corrected gestational age at extubation, weeks & $29.1(25.9-33.0)$ & $29.0(27.3-31.6)$ & 0.98 \\
\hline
\end{tabular}

Northway staging in the group which showed the least response, none of the differences was significant. A multiple linear regression evaluating improvement in the VI at 36-48 hours was performed. No demographic factors, ventilation factors, or radiological features were identified as being significant.

\section{TIME TO EXTUBATION}

The median time to extubation was 6 days. Univariate analyses were performed with the cohort divided into those who extubated in six days or less and those who extubated at seven days or more after the start of dexamethasone (table 3).

Those infants who extubated within a week were more mature $(p=0.0017)$ and consequently of higher birth weight $(\mathrm{p}=0.0310)$ than those infants who failed to extubate within 7 days. There were no significant differences in VI in the first three days of postnatal life between the two groups. Infants who were slower to extubate were more likely to have had radiological evidence of air leak syndromes, with either PIE $(p<0.0001)$ or a pneumothorax requiring an intercostal drain $(\mathrm{p}=0.01689)$, and were also more likely to have Northway stage 2 radiographs $(\mathrm{p}=0.00553)$. Infants who extubated within 7 days had a lower VI before the start of dexamethasone than the infants who were slower to extubate (46.8 vs 60.9 ; $\mathrm{p}$ $=0.0223)$. The corrected gestation at the time of extubation was not significantly different between the two groups. Although there was a

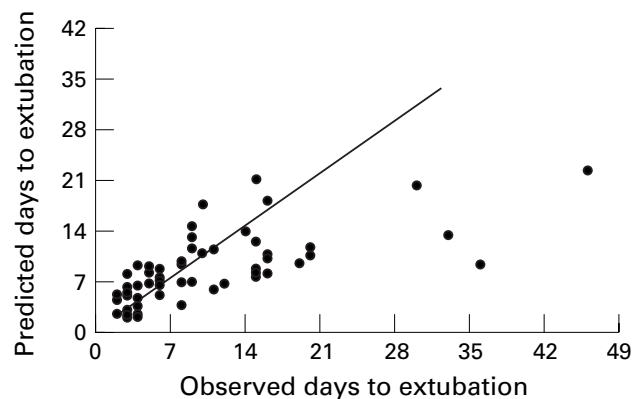

Figure 1 Graph showing regression model of the number of days to extubation from the start of dexamethasone (adjust $R^{2}=0.5126$ ). trend towards an earlier postnatal age at which dexamethasone was begun in those who did not extubate within a week, this was not significant. After dexamethasone had been started, those that extubated before one week had a significantly greater reduction in VI at 36-48 hours $(p<0.0001)$.

A multiple linear regression was performed examining the variables that were significant in the univariate analysis. The days to extubation were log converted to give a normal distribution and regression resulted in three factors remaining significant, giving a regression equation of: $\log 10$ (days to extubation) $=2.779-$ $($ gestation $\times 0.090)+(\mathrm{VI} \times 0.006)+(\mathrm{PIE} \times$ $0.230)$ where gestation is the value in completed weeks $(p<0.0001)$, VI represents the mean VI in the 12 hours preceding dexamethasone $(p=0.0002)$, and PIE is assigned the value of 0 if there is no evidence of PIE and 1 if any degree of PIE was present on the radiograph $(p=0.0011)$. This equation resulted in an $r$ value of 0.733 , with an adjusted $\mathrm{R}^{2}$ of 0.5126 and a standard error of 0.244 (fig 1 ).

DEATH BEFORE EXTUBATION

Five infants died before extubation. Only one infant died of respiratory failure from CLD. Severe intraventricular haemorrhage (IVH) was cited as the cause of death in two of the five infants. One infant with a grade $3 \mathrm{IVH}$ subsequently developed Candida meningitis, and the remaining infant died of necrotising enterocolitis.

\section{Discussion}

This study has highlighted the range of response to dexamethasone of ventilator dependent preterm infants, both in terms of immediate improvement in ventilatory settings and time to extubation. Randomised trials have consistently shown that dexamethasone reduces the duration of ventilatory support in preterm infants. ${ }^{1-8}$ While randomised controlled trials may show benefit for a study population as a group, they do not necessarily identify those individuals in whom the greatest benefit is achieved, nor those for whom the intervention is potentially harmful or futile. Therefore, 
examining a cohort of infants and comparing responses may reveal associations that can later be tested with specific subgroup analysis in the context of large randomised controlled trials.

Could the retrospective nature of this study have introduced errors? The unit policy of early extubation to nasal CPAP did not change over the time period of the study. And while the regime for dexamethasone administration was changed from a 42 day to a 14 day course during the study, the initial dosage remained unchanged. Thus protocol changes over the period of the study were few and unlikely to have influenced the results.

This is the first study to have described individual variation in the response to dexamethasone treatment. On examining immediate response, there were no identifiable factors that were associated with a change in VI at 36-48 hours. However, those who had not significantly improved by this time took much longer to extubate, so response at $36-48$ hours gave some indication of the likely course of the infant. Nevertheless, the range of times to extubation was still very varied-one infant who had an apparently good response at 36-48 hours did not extubate for a further 44 days, and one who did not significantly improve at 36-48 hours extubated two days later.

The more important outcome of time to extubation after dexamethasone was significantly associated with three factors: the infant's gestation; the presence of PIE on the radiograph; and the VI before starting dexamethasone. Dexamethasone is unlikely to help lung tissue that is structurally very immature or in which extensive fibrotic changes have already occurred. When time to extubation was corrected to postconceptional age there were no differences between those that extubated early or late. This suggests that regardless of the degree of underlying lung damage or disease, a certain maturity is necessary for the infant to extubate successfully.

PIE was the major radiographic feature associated with a poor response. One previous study has suggested that a better response was associated with the radiographic appearance of homogeneous opacity rather than cystic changes. ${ }^{12}$ Univariate analysis of our study population suggested that homogeneous opacity was associated with a more prolonged time to extubation. However, this was no longer significant once gestation had been controlled for. The probable mechanism of action of postnatal steroids is through modulation of the inflammatory response to artificial ventilation. Inflammatory cells and mediators have been demonstrated in endotracheal aspirates in infants at risk of developing CLD ${ }^{13-16}$ and have also been shown to decrease with the administration of corticosteroids. ${ }^{17-20}$ There may be additional actions such as decreasing membrane permeability. ${ }^{16} 1820$ PIE has been observed more commonly in infants who have raised neutrophil elastase activity in tracheal lavage aspirate in the early stages of respiratory distress syndrome, ${ }^{21}$ implicating inflammatory mediators in the aetiology of air leak syn- dromes. However, once PIE has occurred dexamethasone seems be of limited benefit.

The degree of ventilatory support that the infant required before dexamethasone was also significantly associated with the time to extubation. Interestingly, the degree of improvement at 36-48 hours was not significantly associated with the VI before dexamethasone, suggesting that even very sick infants could have a wide variation in response, ranging from both a substantial decrease to a worsening of the ventilatory requirement, and that some infants who did not have high ventilation requirements did not necessarily improve dramatically at 48 hours.

This study has described differences in response to postnatal dexamethasone within a cohort of preterm infants and provided a model by which the time to extubation can be partly predicted. In short, immature sick babies with radiological evidence of PIE cannot be expected to extubate rapidly. Taking these factors into account before starting dexamethasone may assist with a decision to institute a potentially harmful treatment, and in assessing the degree of response that is seen in an individual receiving dexamethasone. Estimation of the response may also assist clinicians in counselling parents about the expected duration of ventilatory support that their infant will require. These data highlight the need for more information on whom to start postnatal steroids, and when, and at what dose and duration.

We thank Mr Neill Jones and Dr Philip Beeby for their assistance with the regression model. rial of dexamethasone in respirator-dependent infants with bronchopulmonary dysplasia. Pediatrics 1985;75:106-11.

2 Harkavy KL, Scanlon JW, Chowdhry PK, Grylack LJ. Dexamethasone therapy for chronic lung disease in ventilatorand oxygen-dependent infants: a controlled trial. $\mathcal{F}$ Pediatr 1989;115:979-83.

3 Cummings JJ, D'Eugenio DB, Gross SJ. A controlled trial of dexamethasone in preterm infants at high risk for bronchopulmonary dysplasia. $N$ Engl $\mathscr{f}$ Med 1989;320:1505-10.

4 Kazzi NJ, Brans YW, Poland RL. Dexamethasone effects on the hospital course of infants with bronchopulmonary dysplasia who are dependent on artificial ventilation. Pediatrics 1990;86:722-7.

5 Collaborative Dexamethasone Trial Group. Dexamethasone therapy in neonatal chronic lung disease: an international placebo-controlled. Pediatrics 1991;88:421-7.

6 Yeh TF, Torre JA, Rastogi A, Anyebuno MA, Pildes RS. Early postnatal dexamethasone therapy in premature infants with sever respiratory distress syndrome: a doubleblind, controlled study. $\mathcal{F}$ Pediatr 1990;117:273-82.

7 Sanders RJ, Cox C, Phelps DL, Sinkin RA. Two doses of early intravenous dexamethasone for the prevention of bronchopulmonary dysplasia in babies with respiratory
distress syndrome. Pediatr Res 1994;36:122-8.

8 Durand M, Sardesai S, McEvoy C. Effects of early dexamethasone therapy on pulmonary mechanics and chronic lung disease in very low birth weight infants: a randlung disease in very low birth weight infants: a

9 Werner JC, Sicard RE, Hansen TWR, Solomon E, Cowett RM, Oh W. Hypertrophic cardiomyopathy associated with dexamethasone therapy for bronchopulmonary dysplasia. F Pediatr 1992;120:286-91.

10 Ng PC, Brownlee KG, Dear PR. Gastroduodenal perforation in preterm babies treated with dexamethasone for bronchopulmonary dysplasia. Arch Dis Child 1991;66:1164-6.

11 Northway WH Jr, Rosan OAK, Porter D. Pulmonary disease following respiratory therapy of hyaline membrane disease. Bronchopulmonary dysplasia. $N$ Engl $7 \mathrm{Med}$ 1967;276:357-68.

12 Kothadia JM, O'Shea TM, Auringer ST, Dillard RG. Response to dexamethasone therapy in ventilated preterm infants depends on radiographic subtype of chronic lung infants depends on radiographic

13 Merrit TA, Cochrane CG, Holcomb K, et al. Newborn tracheal aspirate cytology: classification during respiratory distress syndrome and bronchopulmonary dysplasia. $\mathcal{F}$ Pediatr 1981;98:949-56. 
14 Gerdes JS, Yoder MC, Douglas SD, et al. Tracheal lavage and plasma fibronectin: relationship to respiratory distress syndrome and development of
sia. $\mathcal{F}$ Pediatr 1986;108:601-6.

sia. F Pediatr 1986;108:601-6.
15 Mirro R, Armstead W, Leffler C. Increased airway leukotriene levels in infants with severe bronchopulmonary dysplasia. Am F Dis Child 1990;144:160-1

16 Groneck P, Götze-Speer B, Oppermann M, Eiffert H, Speer CP. Association of pulmonary inflammation and increased microvascular permeability during development of bronchopulmonary dysplasia: a sequential analysis of inflammatory mediators in respiratory fluids of high-risk preterm neonates. Pediatrics 1994;93:712-8.

17 Gerdes JS, Harris MC, Polin RC. Effects of dexamethasone and indomethacin on elastase, (1-proteinase inhibitor, and fibronectin in bronchoalveolar lavage fluid from neonates. f Pediatr 1988;113:727-31.
18 Yoder MC, Chua R, Tepper R. Effect of dexamethasone on pulmonary inflammation and pulmonary function of ventilator-dependent infants with bronchopulmonary dysplasia. Am Rev Respir Dis 1991;143:1044-8.

19 Watts CL, Bruce MC. Effect of dexamethasone therapy on fibronectin and albumin levels in lung secretions in infants with bronchopulmonary dysplasia $f$ Pediatr 1992;121:597-607.

20 Groneck P, Ruess D, Götze-Speer B, Speer CP. Effects of dexamethasone on chemotactic activity and inflammatory mediators in tracheobronchial aspirates of preterm infants at risk for chronic lung disease. F Pediatr 1993;122:938-44.

21 Speer CP, Ruess D, Harms K, Herting E, Gefeller O. Neutrophil elastase and acute pulmonary damage in neonates with severe respiratory distress syndrome. Pediatrics 1993;91:794-9. 